\title{
Which is the Best PID Variant for Pneumatic Soft Robots? An Experimental Study
}

\begin{abstract}
This paper presents an experimental study to compare the performance of model-free control strategies for pneumatic soft robots. Fabricated using soft materials, soft robots, have gained research attention in academia as well as in industry during recent years because of their inherent safety in human interaction. However, due to structural flexibility and compliance, the mathematical model of these soft robots is nonlinear with an infinite degree of freedom (DOF). Therefore, the accurate position (or orientation) control and optimization of their dynamic response remains a challenging task. Most of the existing soft robots being used in industrial and rehabilitation applications use model-free control algorithms such as PID. However, to the best of our knowledge, there has been no systematic study on the comparative performance of the model-free control algorithms and their ability to optimize the dynamic response i.e., reduce overshoot and settling time. In this paper, we present comparative performance of several variants of model-free PID-controllers based on extensive experimental results. Additionally, most of the existing work on model-free control in pneumatic soft-robotic literature use manually tuned parameters, which is a time-consuming, labor-intensive task. We present a heuristic-based coordinate descent algorithm to tune the controller parameter automatically. We presented results for both manually tuned, using the Ziegler-Nichols method, and automatic tuning using the proposed algorithm. We used the experimental results to statistically demonstrate that the presented automatic tuning algorithm results in high accuracy. The experiment results show that for soft robots, the PID-controller essentially reduces to the PI controller. This behavior was observed in both manual and automatic tuning experiments; we also discussed a rationale for removing the derivative term.
\end{abstract}

\section{INTRODUCTION}

Soft robotics, i.e., robotic systems using structures based on soft materials (hereafter, simplified as "soft structures") instead of traditional rigid structures, are increasingly gaining attention. The use of soft structures opens up new opportunities to solve problems in traditional rigid robotic systems, but also leads to new challenges. Foremost among these is how to accurately model and control such systems. A soft structure has an infinite degree of freedom, making it impossible to develop as accurate a model as that for a rigid structure. It, in turn, challenges the fine-grained control of the soft structure, especially the tuning of its dynamic responses. It raises serious concerns in many applications, such as rehabilitation, where the fine-grained control of muscles supported by a soft structure is necessary. Another example is in high-speed applications, such as industrial soft robotic grippers; the fine-tuning of dynamic responses is essential.

Soft robotics, being and emerging field, still have limited research literature on the accurate modeling and dynamic response tuning. Vikas et al. [1], [2] proposed a graph-based, model-free framework for controlling the locomotion of soft robots. Calisti et al. [3], [4] proposed control strategies inspired by aquatic life. Both proposals only deal with the coarse-grained locomotion of soft robots, instead of the fine-grained control and dynamic response tuning. Reymundo et al. [5] used a statistical model to control system responses, but this method assumes the model to be linear and predictable for a given range of operations. These assumptions and the absence of a feedback loop can render the system unstable and produce an undesirable response. Frederick et al. [6]-[8] proposed a Finite Element Method (FEM)-based control strategy. This strategy can achieve high accuracy but requires detailed knowledge of the mechanical properties of the soft structure materials. Furthermore, FEM-based control strategies incur high computation costs, which makes real-time execution impractical on embedded processors. One remedy is to run FEM-based control strategies in a feedforward open-loop fashion. But this makes the control prone to error and decreases overall robustness. Marchese et al. [9]-[11] proposed a model-based control strategy to optimize the dynamic responses of soft robots.

Few model-based approaches, to regulate the dynamic response, have also been proposed in the literature. For example, Ni et al. [12] proposed an approach to control the dynamic response of the soft actuator by attaching an external mechanical damper to the body of a soft robot. Similarly, Wei et al. [13] and Li et al. [14] presented a design of soft actuators with particle chambers. These approaches by dissipating excessive kinetic energy by using mechanical components. However, the mechanical overhead causes the system to become bulky and increases the fabrication cost. Luo et al. [15] proposed a Sliding-Mode Controller (SMC) based controller. However, their proposed controller requires careful manual tuning of controller parameter. All of the controllers mentioned-above heavily rely on the mathematical model of the soft-robots and does not adapt to the mechanical variations in the soft-robot. However, soft robots usually suffer from inevitable wear and tear [16] after several usage cycles, which affect the mathematical model of soft robots; therefore, the performance of these controllers degrade.

Due to the constraints of the above control strategies, should the model of soft robots not be given a priori, model-free closed-loop PID controllers become the choice for fine-grained control and dynamic response tuning [17]. This fact is strengthened by the wide adoption and popularity of PID control. Several variants of model-free PID-controller are discussed in literature [18]-[22]. In this paper, we conduct a detailed comparison of these controllers. We re-evaluated the performance of PID variants for soft robots and highlighted their key differences from the PID-controller for rigid robots. The main contributions of this paper can be summarized as:

- Compilation of comprehensive experimental results to 
analyze the comparative performance of the PID controllers, specifically for the soft robots. The paper compares three different types of PID controllers; regular PID, piecewise PID, and fuzzy PID. Along with two different types of parameter tuning algorithm; Ziegler-Nichols for manual tuning and coordinate descent automatic tuning.

- Various general-purpose controllers have been designed for soft robots in literature without much recognition of the uniqueness of soft robots. This work conducts extensive experiments and uses data to show that the dynamical behaviors of soft robots are indeed different.

- Researchers usually intuitively think PID-controllers do not differ much for both rigid-body robots and soft robots. However, we identify a fundamental difference from extensive experiments. To our knowledge, this is the first time that this inherent difference has been recognized. This difference may help researchers design improved controllers for soft robots by leveraging their uniqueness.

- Following the identified inherent differences of PID control for soft and rigid-body robots, six types of dominating PID variants are systematically evaluated and compared on an experimental soft robot platform. The best PID variant is identified, and the rationale of its outstanding performance is also established.

The remainder of this paper is organized as follows. Section II discusses related work on soft robot control. Section III-A presents PID-controller variants along with formulation of coordinate descent algorithm for automatic parameter tuning of PID-controllers. Section IV describes the platform, methodology, and results of the experiment. Section $\mathrm{V}$ concludes the paper.

\section{BACKGROUND AND RELATED WORK}

Recent developments in soft robotics have focused on the design, fabrication, control, and applications of soft robots. In this section, a brief review of recent advances in soft robotics is presented. Table I contains a summary of this review.

Soft robots are being developed as counterparts to traditional rigid mechanical robots, while offering the same functionality as rigid structures, with the added advantage of flexibility. For Example, in conventional robotic systems, linear motion is provided by using linear actuators such as linear DC motors. To provide the same functionality with soft structures, [23], [24] have proposed a design for soft materials based muscles, actuated using pneumatic systems. Similarly, to produce circular or bending motions, soft actuators [25]-[29] have been designed to replace rigid servos. Efforts have also been made to develop soft structures, capable of performing motions in three-dimensional space. Soft robots shaped like octopus tentacles [30], without any rigid joints or links, are capable of maneuvering in three-dimensional space using pneumatic actuation.

One of the fundamental difference between traditional rigid robots and soft robots is the formulation of an accurate mathematical model. Robots consisting of rigid links and joints can be accurately modeled because their motion is deterministic and can be easily predicted. However, soft robots are flexible, which make them highly nonlinear and theoretically require an infinite degree of freedom to model them accurately [31]. Lack of an accurate mathematical model makes it difficult to formulate an accurate control algorithm. Model estimation techniques have been explored to model the soft robots using a linear mathematical model [32]; however, these estimations fail to model the nonlinear dynamics.

Most soft robots use a pneumatic system for actuation. Although pneumatics are an excellent choice for the actuation of soft structures, however, they make the system heavy, bulky, and reduce portability. Efforts have been made to develop alternate actuation principles for soft robots. Of these, the notable are Dielectric Elastomer Actuators (DEA) [33]-[35], which are capable of producing a large actuation when an electric voltage is applied across the actuator. Much work has also been done on the sensing of soft actuators. The use of combustion-based air pumps for soft robot actuation has also been considered [36]. This actuation principle makes the system more compact but reduces the safety of soft robots.

Because of their flexible structure, soft robots require different sensing mechanisms from rigid robots. The compliant structure of soft robots makes it possible to integrate sensory mechanisms as part of the fabrication material or to embed it inside the soft robots itself. Felt et al. [37] proposed using electrically conductive fabric to cover the body of the soft actuator and measuring deformation by measuring changes in the electrical properties of the fabric. Attempts have also been made to embed the sensor inside the soft robots [38]-[42].

A large proportion of the literature on soft robots is on their use in rehabilitation applications [5], [43]-[47]. Traditional rehabilitation robotic systems use rigid exoskeletons to help patients do recovery exercises. Soft robots are more suitable in such applications because of their flexibility, compliance, and human-skin like appearance, which can help to accelerate the recovery process. Object grippers based on soft robots [48] have also been shown to be competitive with rigid robotic structures. These grippers can easily handle objects of various sizes and shapes. Delicate materials are also much more safely handled using soft grippers.

As discussed in Section I, research on the control of soft robots is still in its infancy. The subject has only attracted research attention after recent developments in the design, fabrication, and sensing of new soft actuators. The most common control approach used in soft robots based applications is the closed-loop PID-controller [17] because it does not require an a priori mathematical model of the soft robot. Efforts have also been made to approximate the mathematical model of the soft robot using linear ODEs and to use an adaptive controller to compensate for unmodeled parameters [32]. Other control methods introduced in the literature also require an a priori mathematical model of the soft robot. Since an accurate analytical model for soft robots cannot be formulated, most of the industrial application of the soft robots use model-free control techniques.

\section{PID-CONTROLlERS FOR SOFT RobOTS}

In this section, mathematical formulation of three such PID variant controllers is presented. The variants presented here 
TABLE I: Summary of recent work in soft Robotics.

\begin{tabular}{|c|c|c|c|c|c|}
\hline & $\begin{array}{c}\text { Design \& } \\
\text { Fabrication }\end{array}$ & Sensing & Modelling & Control & Applications \\
\hline Linear actuator [23], [24] & Yes & No & Static \& Dynamic & No & No \\
\hline Bending actuator [25]-[27] & Yes & No & Static & No & No \\
\hline Other actuators [30], [33]-[36] & Yes & No & Static & No & No \\
\hline Linear sensing [37] & No & Yes & Static & No & No \\
\hline Embedded sensing [38], [39] & Yes & Yes & Static & No & Yes $^{1}$ \\
\hline Soft robotic systems [43], [48] & Yes & No & Static & Static & Yes $^{2}$ \\
\hline model-free control [1], [2], [5] & No & No & Static & Static & No \\
\hline Bio-inspired control [3], [4] & Yes & No & Static & Static & No \\
\hline Finite Element Method (FEM) [6] & No & No & Static & Static & No \\
\hline Dynamic Control [9]-[11] & No & No & Static \& Dynamic & Static \& Dynamic & No \\
\hline
\end{tabular}

include Piecewise PID and Fuzzy PID, whcih differ in the way PID parameters adapt with input reference angle. This section also present automatic algorithm to obtain optimal controller parametrs by optimizing the dynamic response of soft robot.

\section{A. Formulating the Controllers}

The following presents the formulation of a regular PID, a piecewise PID, and a fuzzy PID, which are used for comparison in this paper, and discuss their differences.

\section{1) A Regular PID-controller}

Let us denote a state of the soft robot plant as $\theta(t)$, and the desired or reference state as $\theta_{r}$. The system error is then $e(t)=\theta_{r}-\theta(t)$, and the system control input is denoted as $\mathrm{u}(\mathrm{t})$. Based on this notation, a regular PID-controller defines the system input $u(t)$ as

$$
u(t)=K_{p} e(t)+K_{i} \int_{0}^{t} e(\tau) d \tau+K_{d} \frac{d}{d t} e(t),
$$

where $K_{p}, K_{i}$, and $K_{d}$ are controller parameters. These controller parameters can be adjusted to tune the dynamic responses of the system. The above formulation assumes a continuous time system. In case sensor data acquisition and actuation take place in discrete time, (1) becomes

$$
u[n]=K_{p} e[n]+K_{i} \sum_{i=1}^{n} e[i] \Delta t_{i}+K_{d} \frac{\Delta e_{n}}{\Delta t_{n}},
$$

where $n$ is the discrete index number, $e[n]=\theta_{r}-\theta[n]$, $\Delta e_{n}=e[n]-e[n-1]$, and $\Delta t_{n}$ is the time difference between two consecutive sensor readings, i.e. $(\theta[n]$ and $\theta[n-1])$. For simplicity of notation, let us denote the parameter vector and system error vector as

$$
\mathbb{K}=\left[\begin{array}{c}
K_{p} \\
K_{i} \\
K_{d}
\end{array}\right], \quad \mathbb{E}[n]=\left[\begin{array}{c}
e[n] \\
\sum_{i=1}^{n} e[i] \Delta t_{i} \\
\Delta e_{n} / \Delta t_{n}
\end{array}\right] .
$$

Using this, Equation (2) becomes:

$$
u[n]=\mathbb{K}^{T} \mathbb{E}[n] .
$$

The schematic diagram of the PID-controller used in our experimental system is shown in Fig. 1. The main task in PID control is to adjust vector $\mathbb{K}$ to obtain the desired dynamic responses. The algorithms to tune $\mathbb{K}$ will be explained later.

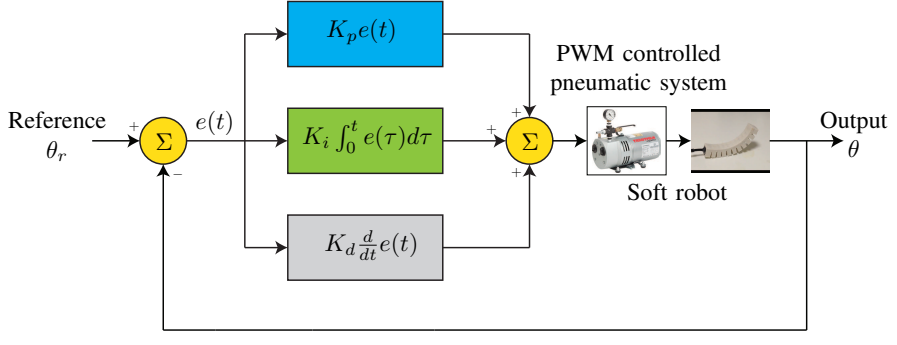

Fig. 1: Schematic diagram of canonical form of PID-controller for soft muscle control.

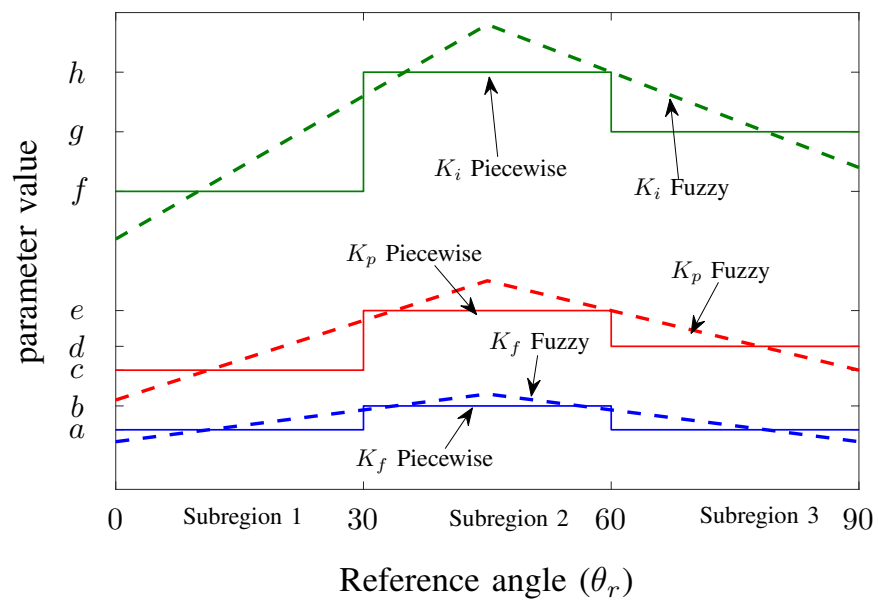

Fig. 2: Parameter variation in a Piecewise PID (solid lines) vs. a Fuzzy PID (dashed lines).

\section{2) Piecewise PID-controller}

The regular PID-controller of Equations (2) and (3) fixes the control parameter vector $\mathbb{K}$ for all possible values of reference input $\theta_{r}$. However, to fine tune dynamic responses, different $\theta_{r} \mathrm{~s}$ needs different optimal control parameters. In other words, the optimal $\mathbb{K}$ is dependent on the given $\theta_{r}$. A piecewise PID matches this demand by expressing $\mathbb{K}$ as a piecewise constant function of $\theta_{r}$. The valid range of $\theta_{r}$ is divided into several subranges and the value of $\mathbb{K}$ is separately tuned for each subrange. That is, $\mathbb{K}$ now becomes $\mathbb{K}\left(\theta_{r}\right)$. Correspondingly, Formula 3 becomes

$$
u[n]=\mathbb{K}\left(\theta_{r}\right)^{T} \mathbb{E}[n] .
$$

Fig. 2 illustrates the concept of the piecewise PID control parameter vector $\mathbb{K}$. 


\section{3) Fuzzy PID-controller}

Although a piecewise PID increases flexibility in tuning control parameter vector $\mathbb{K}$, abrupt changes still occur at the boundaries of the subranges (see Fig. 2). Fuzzy PID further increases the flexibility if the controller parameters $\mathbb{K}$. Instead of defining $\mathbb{K}\left(\theta_{r}\right)$ as a piecewise constant function, it is defined $\mathbb{K}\left(\theta_{r}\right)$ as a piecewise linear and continuous function of $\theta_{r}$. This formulation allows for a continuous change in the control parameter vector $\mathbb{K}$ at the boundaries of the subranges. The concept of the fuzzy control parameter vector is shown in Fig. 2. The mathematical formulation of the Fuzzy PID-controller is the same as in Equation (4); the only difference is how $\mathbb{K}$ changes with $\theta_{r}$.

\section{B. Control Parameter Tuning}

After formulation, the second task in implementing the PID-controllers as mentioned earlier is to tune the control parameter vector $\mathbb{K}$, for fine-grained control or optimal dynamic responses. Two methods to tune $\mathbb{K}$ will be discussed. The first is the manual method, which involves visually observing the performance of the system with different values of $\mathbb{K}$ and selecting an optimal value. The second method is to tune $\mathbb{K}$ using algorithms automatically. Both of these tuning methods are explained below.

\section{1) Manual Tuning}

Manual tuning involves adjusting the control parameter vector $\mathbb{K}$ by hand and observing the corresponding system performances. If the performances are undesirable, then $\mathbb{K}$ should be changed manually, and the performances observed again, and so on. In this way, by manually adjusting $\mathbb{K}$ in a hit-and-miss approach, it is possible to achieve the desired system performances. This approach labor-intensive and depends heavily on the experience and judgment of the operators.

We used the Ziegler-Nichols method to manually tune the PID parameters which goes as follow: given $\theta_{r}$, we first set $K_{i}$ and $K_{d}$ to zero and increase $K_{p}$ until the step response of the control system is in converging oscillations. If the steady-state response contains a constant error, then $K_{p}$ should be kept constant and $K_{i}$ increased until the steady-state response error decreases to zero. At this stage, we can further fine-tune the oscillations behavior of the step response: settling time and overshoot. For example, to reduce overshooting, $K_{i}$ should be kept constant, and $K_{d}$ should be increased.

\section{2) Automatic Tuning}

In contrast to manual tuning, automatic tuning is more principled and is based on rigorous algorithms. Several metaheuristic optimization algorithms have been proposed in the literature to tune the controller parameter [49]-[51]. However, most of these algorithms assume that the mathematical model of the controlled system is known apriori. However, in the case of soft robots, such assumptions are not practical. Therefore, we need to rely on the real robot to obtain the response and calculate the performance metric. Therefore, we used a classic automatic tuning algorithm, called Coordinate Descent Algorithm [52]. It is

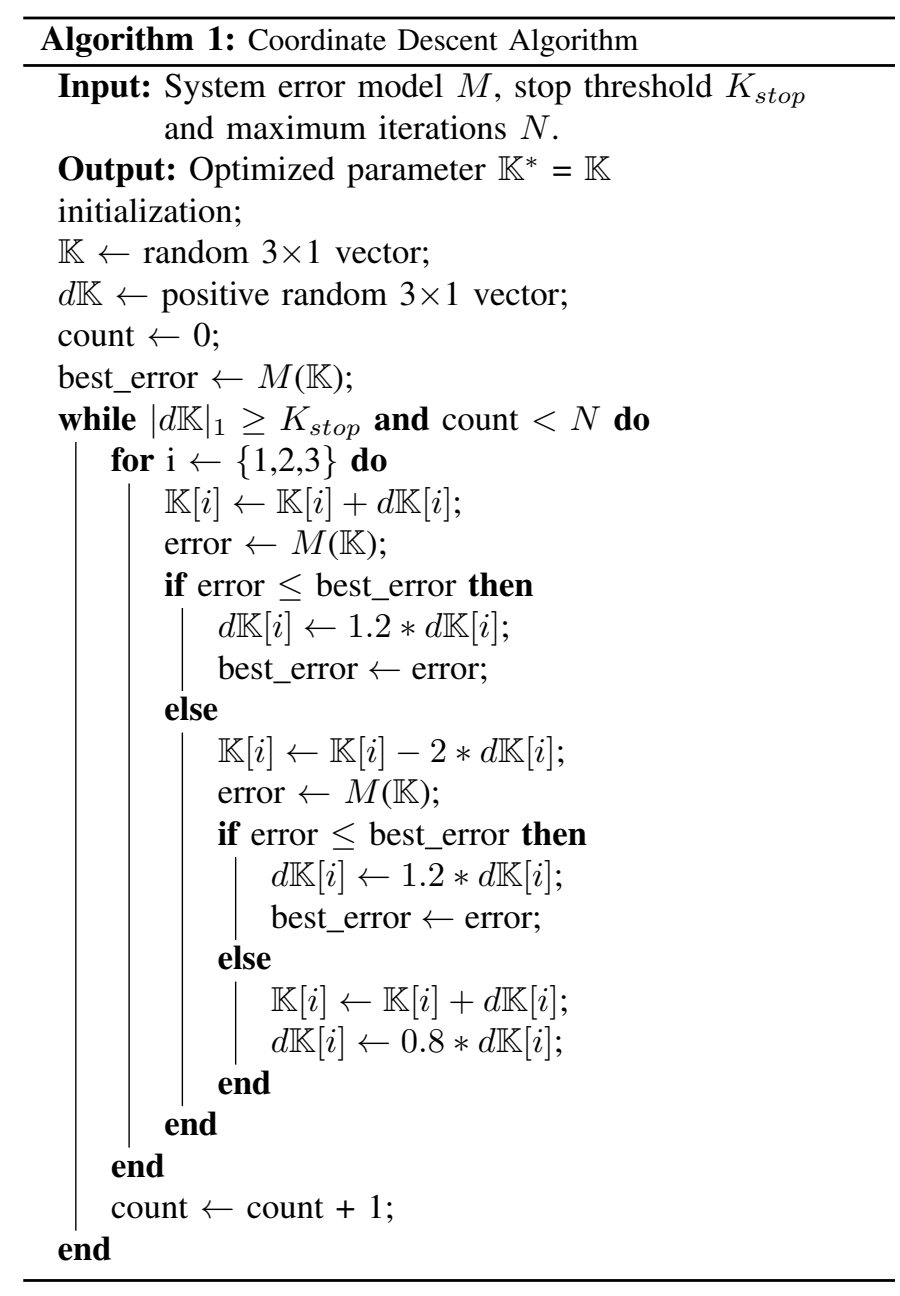

a metaheuristic algorithm that tries to optimize a concerned control performance metric in the output of the system. The pseudo-code is given in Algorithm 1. The heuristics of the algorithm runs as follows. It starts with a random value for the controller parameter vector $\mathbb{K}$ and a small probing positive step size $d \mathbb{K}$. It then calculates concerned performance metric $M(\mathbb{K})$ of the control system. If the metric is not good enough, then the algorithm probes the neighborhood of $\mathbb{K}$ with a step size of $d \mathbb{K}$. If the metric improves, we update $\mathbb{K}$ and increase the step size $d \mathbb{K}$; otherwise, we try smaller step sizes. We repeat the above process until the step size shrinks below a threshold or until a maximum iteration count is reached.

Note that the Coordinate Descent Algorithm is a metaheuristic algorithm; therefore, it may converge to a local optimum instead of a global optimum, or not converge at all, depending on the initial value. Also, in this paper, our concerned control performance metric is defined by the following:

$$
M(\mathbb{K})=\frac{1}{n} \sum_{i=1}^{n}\left(|e[i]|+\left|\frac{\Delta e_{i}}{\Delta t_{i}}\right|\right),
$$

where $e[i]$ and $\Delta e_{i} / \Delta t_{i}$ are same as defined in (2). This definition takes into consideration both; the response oscillation magnitude (as measured by $|e[i]|$ ) and the oscillation slope (as measured by $\left.\left|\Delta e_{i} / \Delta t_{i}\right|\right)$, and therefore 


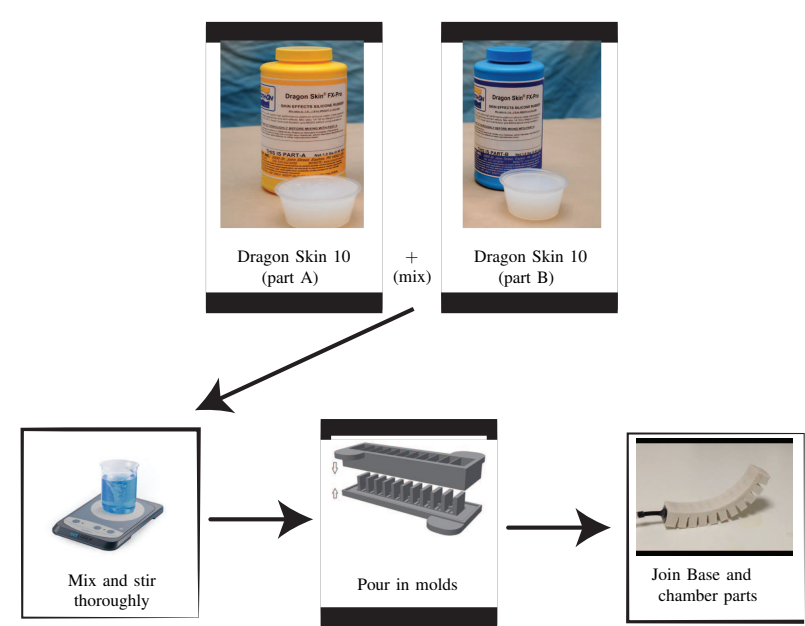

Fig. 3: Flowchart of the process for fabricating the PneuNet used in our experimental platform.

can reflect the control accuracy and dynamic response quality.

To summarize, our Coordinate Descent Algorithm aims to find the optimal PID control parameter vector

$$
M^{*}=\underset{\mathbb{K}}{\operatorname{argmin}} M(\mathbb{K}),
$$

by minimizing the $M(\mathbb{K})$ of Equation 5, which is a holistic metric of control accuracy and dynamic response quality.

\section{EXPERIMENTS AND COMPARISON}

In this section, we evaluate the various model-free closed-loop PID-controllers on soft robots via experiments.

\section{A. Experimental Platform}

PneuNet [25] is a soft bending robot, fabricated using silicones. It consists of a series of chambers connected through a central channel. One side of each chamber is stiffer than the other. This difference in stiffness causes PneuNet to bend when chambers are actuated using air pressure. The fabrication, sensing, and actuation mechanisms are described now.

\section{1) Fabrication}

The 3D designs of PneuNets molds are open source and available in the Soft Robotic Toolkit [53]. The silicone material used for fabrication is Dragon Skin 10 from SMOOTH-ON Inc. [54]. This silicone material consists of two separate mixtures named Part A and Part B. The process of curing the silicone starts after both mixtures are combined thoroughly and left in the open air for some time.

We 3D printed the molds and prepared the elastomer by thoroughly mixing Part A and Part B of Dragon Skin 10 in a 1:1 ratio by volume. The printed molds consist of three parts: one part is used to fabricate the bottom of PneuNet, and the other two parts are combined to fabricate the upper chambers. A prepared elastomer is poured into the molds and allowed to be cured in the open air at room temperature for 8 hours. Since the bottom of the actuator needs to be stiffer, a piece of paper is embedded inside it. Once the bottom and upper chambers are cured, they are glued with a liquid elastomer to form a holistic PneuNet soft robot. Fig. 3 shows the whole fabrication process.

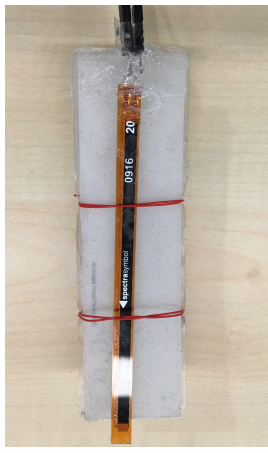

(a)

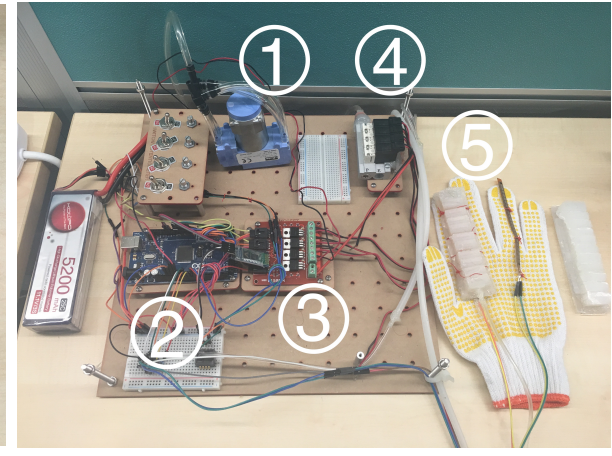

(b)
Fig. 4: (a) Sensing mechanism in experiments to measure the bending angle of the PneuNet (PneuNet: white object, flex sensor: brown strip). (b) Experimental platform: containing (1) an Air pump, (2) a Arduino Mega, (3) MOSFET switches, (4) valves, (5) the bending sensor and the PneuNet robot.

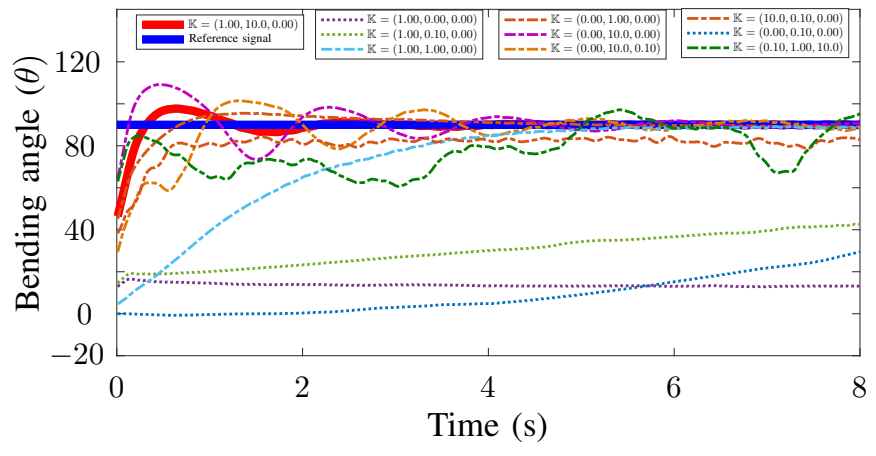

Fig. 5: System responses for the manual tuning of control parameters $\mathbb{K}$ for $\theta_{r}=90^{\circ}$. The solid blue line represents the reference response while solid red line represents the response, visually chosen to be the best.

\section{2) Sensing and Actuation}

PneuNets are capable of producing only angular motion. Therefore, FlexiForce bending sensors are used to determine the angular motion of PneuNets. Such a sensor is a resistor, whose resistance changes with the bending angle. We developed mapping from resistance readings to bending angles through field measurements. The sensor is then attached to the base of our PneuNet soft robot, as shown in Fig. 4a.

We used a $12 \mathrm{~V}$ DC air pump to actuate our PneuNet soft robot. The pump was connected to the robot via an electro-mechanic valve controlled by a MOSFET switch. The valve has a response period of $T=1 / 30(\mathrm{~s})$, and an adjustable switched-on time duration $T_{o}(\mathrm{~s})$ in each response period. The ratio $T_{o} / T$ is called the duty cycle. By adjusting the MOSFET switch duty cycle, we can adjust the open/close time ratio of the valve, and in turn, control the air pressure injected into the PneuNet soft robot. Between the bending sensor and the MOSFET switch is the PID-controller that is under evaluation. The controller runs on an Arduino Mega 2560 board and outputs PWM signals to adjust the duty cycle of the MOSFET switch. The developed system is shown in Fig. 4b.

\section{B. Experiments, Results \& Discussion}

We conducted a series of step and sinusoidal input experiments to demonstrate the effectiveness of tuning and 


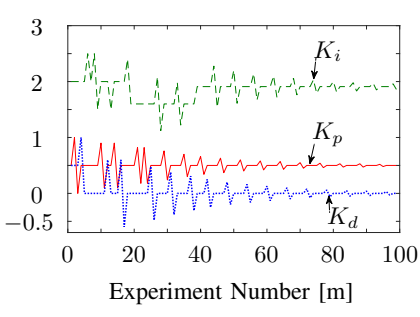

(a)

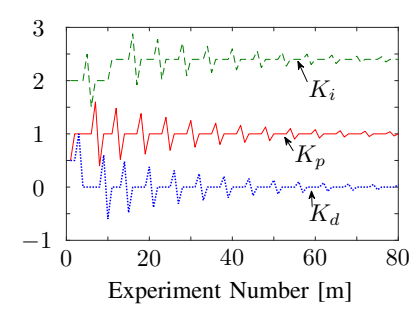

(b)

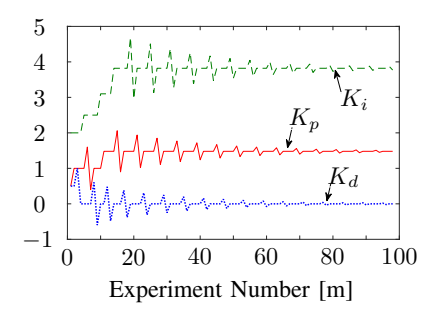

(c)

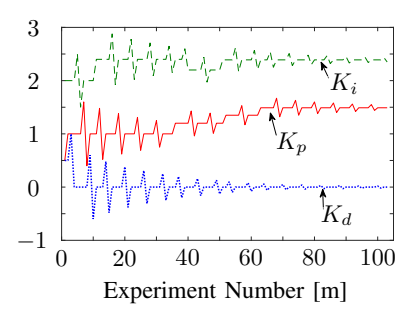

(d)

Fig. 6: Control parameter convergance by coordinate descent. Each iteration of coordinate descent represents one expreiment. (a) shows the tuning results for the complete operation range $\theta_{r} \in\left[0^{\circ}, 90^{\circ}\right]$ i.e., $\theta_{r}$ is sequentially selected from set $\left\{15^{\circ}, 45^{\circ}, 75^{\circ}, 90^{\circ}\right\}$. The final values are $K_{p}=0.5, K_{i}=1.86$, and $K_{d}=0$. (b) shows the results for subrange $\theta_{r} \in\left[0^{\circ}, 30^{\circ}\right)$ i.e., $\theta_{r}=15^{\circ}$, the final values are $K_{p}=1, K_{i}=2.44$, and $K_{d}=0$. (c) shows the results for subrange $\theta_{r} \in\left[30^{\circ}, 60^{\circ}\right)$ i.e., $\theta_{r}=45^{\circ}$, the final values are $K_{p}=1.48, K_{i}=3.77$, and $K_{d}=0$. (d) shows the results for subrange $\theta_{r} \in\left[60^{\circ}, 90^{\circ}\right)$ i.e., $\theta_{r}=75^{\circ}$, the final values are $K_{p}=1.49, K_{i}=2.43$, and $K_{d}=0$.

TABLE II: Summary of the parameter tuning experiments. The final tuned values are given.

\begin{tabular}{ccccccc}
\hline \hline \multirow{2}{*}{$\theta_{r}$} & \multicolumn{3}{c}{ Manual Tuning } & \multicolumn{3}{c}{ Automatic Tuning } \\
& $K_{p}$ & $K_{i}$ & $K_{d}$ & $K_{p}$ & $K_{i}$ & $K_{d}$ \\
\hline 15 & 1 & 1 & 0 & 1 & 2.44 & 0 \\
45 & 1 & 2 & 0 & 1.48 & 3.77 & 0 \\
75 & 1 & 5 & 0 & 1.49 & 2.43 & 0 \\
\hline \hline
\end{tabular}

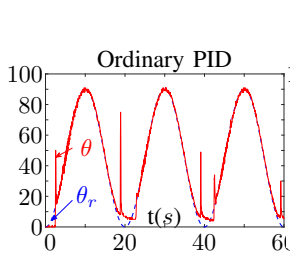

(a)

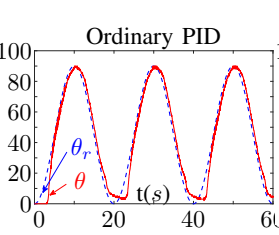

(d)
Manual Tuning

(b)

Automatic Tuning

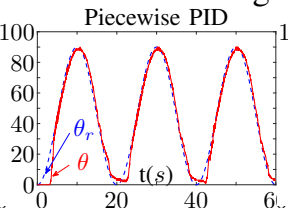

(e)

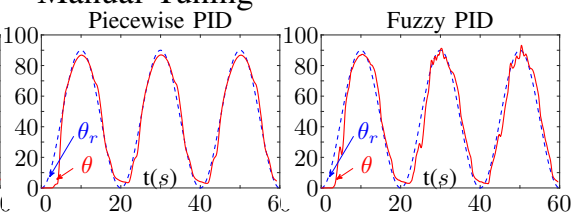

(c)

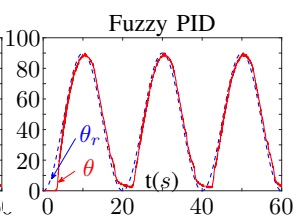

(f)
Fig. 7: Response of one trial (out of 25) for six distinct methods with a sinusoidal reference signal of $0.05 \mathrm{~Hz}$. At low frequencies, the controller can accurately track time varying reference signals, hence the low value of the error metric (7).

control algorithms, respectively. First, the results for the parameter tuning experiments, both manual and automatic, will be presented. The tuned parameters are then used to compare the accuracy of the presented controllers.

\section{1) Parameter Tuning}

To use the piecewise and fuzzy PID formulations described in Section III-A, the entire reference angle range $\left[0^{\circ}, 90^{\circ}\right]$ is divided into the following three subranges: $\left[0^{\circ}, 30^{\circ}\right)$, $\left[30^{\circ}, 60^{\circ}\right)$ and $\left[60^{\circ}, 90^{\circ}\right]$. The midpoint of each subrange, i.e. $\left(15^{\circ}, 45^{\circ}\right.$, and $\left.75^{\circ}\right)$ is used as the representative point when tuning the control parameter for that subrange. The endpoints of the entire range, i.e., $0^{\circ}$ and $90^{\circ}$, are also considered for tuning. Since $0^{\circ}$ is trivial $\left(\theta_{r}=0^{\circ}\right.$ is the natural state of the PneuNet), the results are only presented for $\theta_{r}=90^{\circ}$.

In manual control parameter tuning experiments, the PneuNet is given a step input of $\theta_{r}=90$ while manually adjusting control parameter vector $\mathbb{K}$. The observed responses are shown in Fig. 5. The blue line shows the desired response, whereas the solid red line shows the response that was chosen as the best by the observer because of the short rising time and damped oscillations after the reference angle was reached. In all of the cases, it is observed that setting $K_{d} \neq 0$ results in oscillations and unstable responses, therefore $K_{d}=0$ is an optimal value for the PneuNet actuation. It can be explained in the context of the interpretation of PID-controller terms. The derivative term in PID is essentially a prediction of future behavior of the robotic system. Estimation of the derivative term is trivial for a rigid robotic system since its behavior is mathematically predictable and strictly follow system model. In contrast, soft robots are characterized by a high degree of uncertainty and chaotic motion. Therefore, the estimation of future motion usually results in large variations and errors. These variations manifest themselves in the form of unexpected variation in controller output, thus resulting in erratic behavior. Therefore, a combination of $K_{p} \neq 0$ and $K_{i} \neq 0$ is recommended since they can produce the desired performance. This behavior can also be explained in term of the second-order lumped element model of the soft robot. By analyzing a general second-order model of a robotic system driven by a PID controller, the system can become unstable if the natural damping of the robot is very small. Since soft robots are inherently flexible, therefore, the internal mechanical damping produced by the elastic material is minimal. Thus using a large value of $K_{d}$ can render the system unstable. This heuristic was used while manually adjusting the control parameters for $\theta_{r}=15^{\circ}, 45^{\circ}, 75^{\circ}$. Table II summarizes the results of the manual tuning experiments.

In automatic parameter tuning experiments, coordinate descent algorithm described in Section III-B is used to minimize performance metric (5). The PneuNet is programmed to sequentially select the reference angle from the set $\left\{15^{\circ}, 45^{\circ}, 75^{\circ}, 90^{\circ}\right\}$ for an equal amount of time. Fig. 6a shows the convergence of control parameters $\mathbb{K}$ in 96 iterations of the coordinate descent. For the described experiment, the set of parameters that produces the minimum value for a performance metric (5), is chosen as the best. These results also support the assertion that $K_{d}=0$ is an optimal value for the soft actuator.

The convergence of the control parameters for the first 
TABLE III: Comparison between 6 differnet scenarios of PID variants and tuning algorithm is summarized. For each scenario, 25 experiments are performed and summarized as median along with first and third qurantiles of error metric defined in (7). In most cases Fuzzy PID with automatic tuning produce best results.

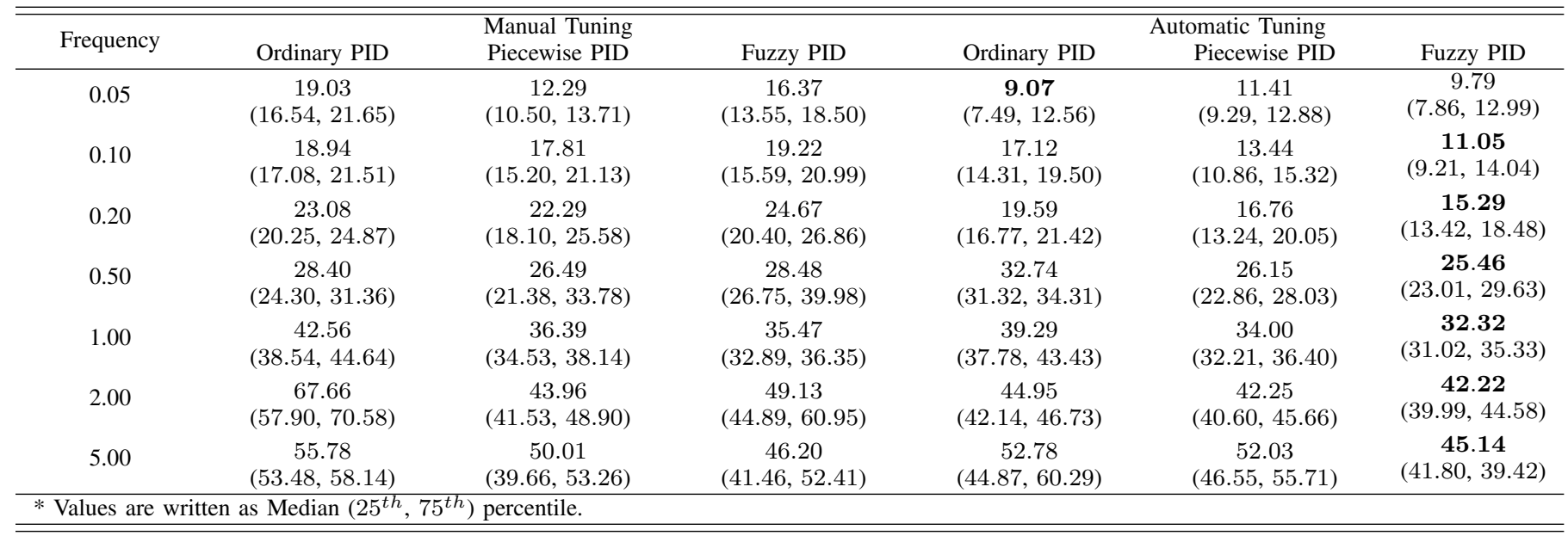

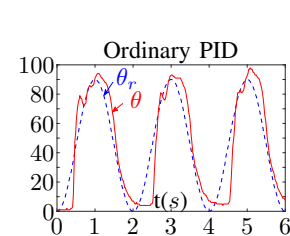

(a)

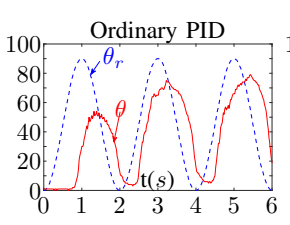

(d)

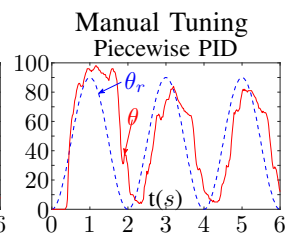

(b)

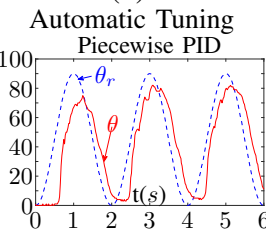

(e)

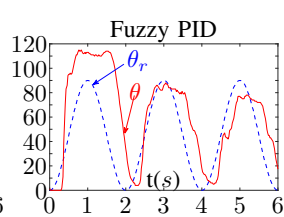

(c)

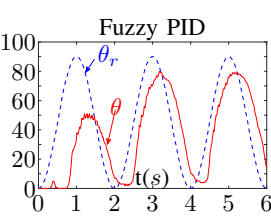

(f)

Fig. 8: Response of one trial (out of 25) for six distinct methods with a sinusoidal reference signal of $0.5 \mathrm{~Hz}$. At increase in frequency of above $0.05 \mathrm{~Hz}$ the output begins to lag the input due to the slow reposne of the PneuNet. The error metric of (7) is high as compared to the $0.05 \mathrm{~Hz}$.

subrange $\left[0^{\circ}, 30^{\circ}\right)$ i.e. $\theta_{r}=15^{\circ}$, using the coordinate descent is shown in Fig. 6b. Similar results are shown for the second subrange $\left[30^{\circ}, 60^{\circ}\right)\left(\theta_{r}=45^{\circ}\right)$ in Fig. $6 \mathrm{c}$ and for the third subrange $\left[60^{\circ}, 90^{\circ}\right)\left(\theta_{r}=75^{\circ}\right)$ in Fig. $6 \mathrm{~d}$. These results further strengthen the assertion that $K_{d}=0$ is optimal. Table II gives a summary of the parameter tuning experiments.

\section{2) Comparison of Controllers}

The following presents a comparison of the three PID-controllers, presented in Section III-A, with manual and automatic tuned control parameters. The controllers are applied with sinusoidally varying reference signals of different frequencies. The controllers are compared based on their ability to accurately track time-varying reference signals. The tracking accuracy of a controller is calculated using the following error metric:

$$
E=\frac{1}{n} \sum_{i=1}^{n}\left|\theta_{r}[i]-\theta[i]\right|
$$

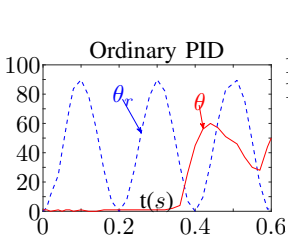

(a)

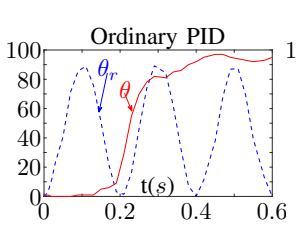

(d)

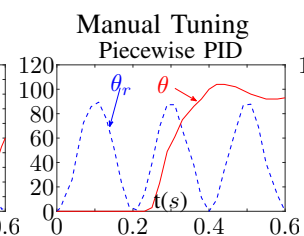

(b)

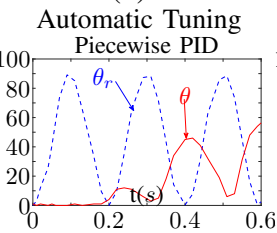

(e)

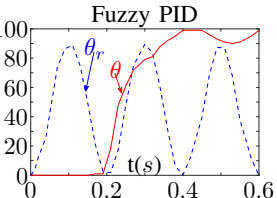

(c)

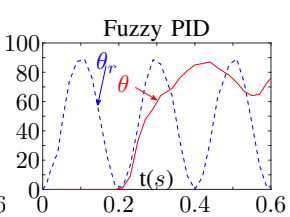

(f)
Fig. 9: Response of one trial (out of 25) for six distinct methods with a sinusoidal reference signal of $5 \mathrm{~Hz}$. At such a high frequency, the physical property (e.g. inertia) of soft muscles constraint the frequency at which it can track input sinusoidal reference. The soft muscle is completely unable to track the reference signal.

where $\theta_{r}[i]$ is a sinusoidally varying reference angle and $\theta[i]$ is the PneuNet bending angle from the sensor.

Comparisons between the following six distinct PID controllers: $\{$ Ordinary, Piecewise, Fuzzy $\}$ PID $\times\{$ Manual, Automatic $\}$ is discussed now. The tuned control parameters summarized in Table II are used to perform all of the experiments. Each method was tested using seven reference signal frequencies of progressively increasing distinct values, varying from $0.05 \mathrm{~Hz}$ to $5 \mathrm{~Hz}$. A total of 25 trials were performed for each experimental case. The response of one trial (out of 25) on tracking the signal frequency of $0.05 \mathrm{~Hz}$ is shown in Fig. 7. Due to the low frequency of the reference signal, the PneuNet can accurately track the time-varying reference angle. Statistics on the error distributions in the 25 trials for each experiment are shown in Fig. 10. It can be seen that in most cases, the automatically tuned parameters usually provided better results than the manually tuned parameters. A similar trend can be observed for the Piecewise and Fuzzy PID-controllers, i.e., the error metric is of a smaller value as compared to the Ordinary 


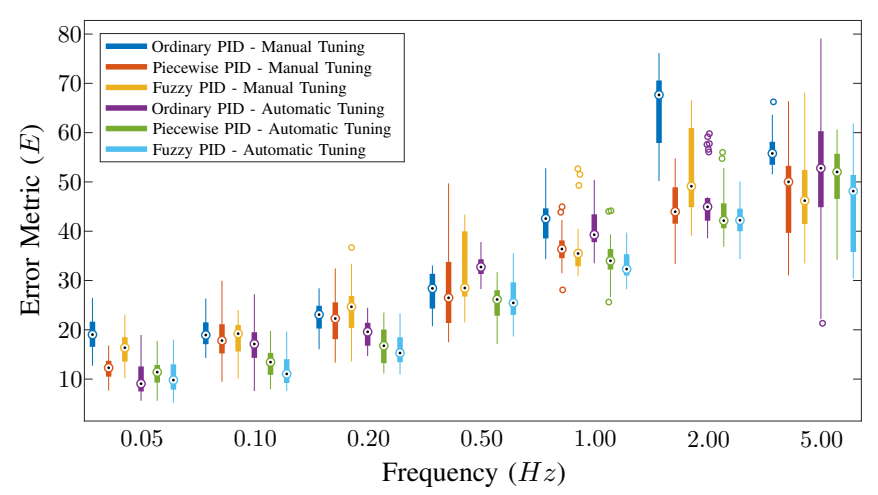

Fig. 10: Summary of the tracking error comparision of six distinct experiments at frequencies ranging from $0.05 \mathrm{~Hz}$ to $5 \mathrm{~Hz}$. The dots shows the median value while box edges shows $25^{t h}$ and $75^{t h}$ percentile. At higher frequencies, the error metric defined by (7) increase.

PID. These observations are consistent with the theory of PID-controllers. Since Piecewise and Fuzzy PID-controllers continually adapt the parameters according to the reference angle, they provide superior performance as compared to the ordinary PID-controller. At very high frequency, the performance deteriorates significantly; therefore, in that fuzzy might exhibit high error as compared to other methods. Similarly, at low frequency, the system can easily track reference signal; therefore, all methods produce a similar performance.

Then moving toward higher frequency in Fig. 10, it can be observed that the error metric began to increase gradually. It can be attributed to the quickly varying reference angle and the slow response rate of the PneuNet. The mechanical properties of the PneuNet (i.e., inertia and the response rate of hydraulic systems) contribute to the slow response, in the case of a sudden change in reference angle. From the experiments, it was observed that the PneuNet could track reference signals of up to $0.5 \mathrm{~Hz}$. Higher frequencies will cause error metrics of large value. The response in one trial (out of 25) for a reference signal frequency of $0.5 \mathrm{~Hz}$ is shown in Fig. 8. In this case, the PneuNet is barely able to track the reference signal, with a large lag between output and input. Similar results for $5 \mathrm{~Hz}$ frequency are shown in Fig. 9.

\section{CONCLUSION}

In this paper, we presented a comparison between three different PID-based control algorithms to operate a soft robot. Since PID-controllers require control parameter tuning, we presented a brief overview of manual and automatic control parameter tuning algorithms. We showed that an automatic tuning algorithm such as a coordinate descent could be used to optimize a defined performance metric to obtain optimal control parameters. A comparison was made between six distinct methods. It was shown that automatically tuned parameters could generally produce better results than manually tuned parameters. Furthermore, the Piecewise and Fuzzy PID was shown to be more accurate in most cases because of their flexibility in continually adapting the control parameters based on a reference angle. Before ending this section as well as this paper, it is worth mentioning that this is the first systematical evaluation of various PID-controller variants use extensive data from real experiments and the first time that identifies a critical difference between rigid-body and soft robots.

\section{REFERENCES}

[1] V. Vikas, P. Grover, and B. Trimmer, "Model-free control framework for multi-limb soft robots," in Intelligent Robots and Systems (IROS), 2015 IEEE/RSJ International Conference on, pp. 1111-1116, IEEE, 2015.

[2] H.-T. Lin, G. G. Leisk, and B. Trimmer, "Goqbot: a caterpillar-inspired soft-bodied rolling robot," Bioinspiration \& biomimetics, vol. 6, no. 2, p. 026007, 2011.

[3] M. Calisti, M. Giorelli, G. Levy, B. Mazzolai, B. Hochner, C. Laschi, and P. Dario, "An octopus-bioinspired solution to movement and manipulation for soft robots," Bioinspiration \& biomimetics, vol. 6, no. 3, p. 036002, 2011.

[4] A. Marchese, C. Onal, and D. Rus, "Autonomous soft robotic fish capable of escape maneuvers using fluidic elastomer actuators," Soft Robotics, vol. 1, no. 1, pp. 75-87, 2014.

[5] P. Polygerinos, S. Lyne, Z. Wang, L. Nicolini, B. Mosadegh, G. Whitesides, and C. Walsh, "Towards a soft pneumatic glove for hand rehabilitation," in Intelligent Robots and Systems (IROS), 2013 IEEE/RSJ International Conference on, pp. 1512-1517, IEEE, 2013.

[6] F. Largilliere, V. Verona, E. Coevoet, M. Sanz-Lopez, J. Dequidt, and C. Duriez, "Real-time control of soft-robots using asynchronous finite element modeling," in Robotics and Automation (ICRA), 2015 IEEE International Conference on, pp. 2550-2555, IEEE, 2015.

[7] F. Faure, C. Duriez, H. Delingette, J. Allard, B. Gilles, S. Marchesseau, H. Talbot, H. Courtecuisse, G. Bousquet, I. Peterlik, et al., "Sofa: A multi-model framework for interactive physical simulation," in Soft Tissue Biomechanical Modeling for Computer Assisted Surgery, pp. 283-321, Springer, 2012.

[8] C. Duriez, "Control of elastic soft robots based on real-time finite element method," in Robotics and Automation (ICRA), 2013 IEEE International Conference on, pp. 3982-3987, IEEE, 2013.

[9] A. Marchese, R. Tedrake, and D. Rus, "Dynamics and trajectory optimization for a soft spatial fluidic elastomer manipulator," The International Journal of Robotics Research, vol. 35, no. 8, pp. 1000-1019, 2016.

[10] F. Renda, M. Giorelli, M. Calisti, M. Cianchetti, and C. Laschi, "Dynamic model of a multibending soft robot arm driven by cables," IEEE Transactions on Robotics, vol. 30, no. 5, pp. 1109-1122, 2014.

[11] I. Gravagne, C. Rahn, and I. Walker, "Large deflection dynamics and control for planar continuum robots," IEEE/ASME transactions on mechatronics, vol. 8, no. 2, pp. 299-307, 2003.

[12] F. Ni, A. Henning, K. Tang, and L. Cai, "Soft damper for quick stabilization of soft robotic actuator," in Real-time Computing and Robotics (RCAR), IEEE International Conference on, pp. 466-471, IEEE, 2016.

[13] Y. Wei, Y. Chen, T. Ren, Q. Chen, C. Yan, Y. Yang, and Y. Li, "A novel, variable stiffness robotic gripper based on integrated soft actuating and particle jamming," Soft Robotics, vol. 3, no. 3, pp. 134-143, 2016.

[14] Y. Li, Y. Chen, T. Ren, and Y. Hu, "Passive and active particle damping in soft robotic actuators," in Robotics and Automation (ICRA), 2018 IEEE International Conference on, pp. 1547-1552, IEEE, 2018.

[15] M. Luo, E. H. Skorina, W. Tao, F. Chen, S. Ozel, Y. Sun, and C. D. Onal, "Toward modular soft robotics: Proprioceptive curvature sensing and sliding-mode control of soft bidirectional bending modules," Soft robotics, vol. 4, no. 2, pp. 117-125, 2017.

[16] S. Terryn, J. Brancart, D. Lefeber, G. Van Assche, and B. Vanderborght, "Self-healing soft pneumatic robots," Sci. Robot., vol. 2, p. eaan4268, 2017.

[17] G. Gerboni, A. Diodato, G. Ciuti, M. Cianchetti, and A. Menciassi, "Feedback control of soft robot actuators via commercial flex bend sensors," IEEE/ASME Transactions on Mechatronics, 2017.

[18] K. Ang, G. Chong, and Y. Li, "Pid control system analysis, design, and technology," IEEE transactions on Control Systems Technology, vol. 13, no. 4 , pp. 559-576, 2005.

[19] K. J. Åström and T. Hägglund, PID controllers: theory, design, and tuning, vol. 2. Instrument society of America Research Triangle Park, NC, 1995.

[20] K.-S. Tang, K. F. Man, G. Chen, and S. Kwong, "An optimal fuzzy pid controller," IEEE Transactions on Industrial Electronics, vol. 48, no. 4, pp. 757-765, 2001. 
[21] X. Luo, M. Zhou, S. Li, Y. Xia, Z.-H. You, Q. Zhu, and H. Leung, "Incorporation of efficient second-order solvers into latent factor models for accurate prediction of missing qos data," IEEE transactions on cybernetics, vol. 48, no. 4, pp. 1216-1228, 2017.

[22] X. Luo, M. Zhou, S. Li, and M. Shang, "An inherently nonnegative latent factor model for high-dimensional and sparse matrices from industrial applications," IEEE Transactions on Industrial Informatics, vol. 14, no. 5, pp. 2011-2022, 2017.

[23] B. Tondu, "Modelling of the mckibben artificial muscle: A review," Journal of Intelligent Material Systems and Structures, vol. 23, pp. 225-253, 2012.

[24] M. Doumit, A. Fahim, and M. Munro, "Analytical modeling and experimental validation of the braided pneumatic muscle," IEEE transactions on robotics, vol. 25, no. 6, pp. 1282-1291, 2009.

[25] B. Mosadegh, P. Polygerinos, C. Keplinger, S. Wennstedt, R. Shepherd, U. Gupta, J. Shim, K. Bertoldi, C. Walsh, and G. Whitesides, "Pneumatic networks for soft robotics that actuate rapidly," Advanced Functional Materials, 2013.

[26] K. Galloway, P. Polygerinos, C. Walsh, and R. Wood, "Mechanically programmable bend radius for fiber-reinforced soft actuators," in Advanced Robotics (ICAR), 2013 16th International Conference on, pp. 1-6, IEEE, 2013.

[27] Y. Yang, Y. Chen, Y. Li, M. Z. Chen, and Y. Wei, "Bioinspired robotic fingers based on pneumatic actuator and 3d printing of smart material," Soft robotics, vol. 4, no. 2, pp. 147-162, 2017.

[28] X. Luo, J. Sun, Z. Wang, S. Li, and M. Shang, "Symmetric and nonnegative latent factor models for undirected, high-dimensional, and sparse networks in industrial applications," IEEE Transactions on Industrial Informatics, vol. 13, no. 6, pp. 3098-3107, 2017.

[29] X. Luo, M. Zhou, S. Li, Z. You, Y. Xia, and Q. Zhu, "A nonnegative latent factor model for large-scale sparse matrices in recommender systems via alternating direction method," IEEE transactions on neural networks and learning systems, vol. 27, no. 3, pp. 579-592, 2015.

[30] R. Martinez, J. Branch, C. Fish, L. Jin, R. Shepherd, R. Nunes, Z. Suo, and G. Whitesides, "Robotic tentacles with three-dimensional mobility based on flexible elastomers," Advanced Materials, vol. 25, no. 2 , pp. 205-212, 2013.

[31] C. Della Santina, R. K. Katzschmann, A. Bicchi, and D. Rus, "Dynamic control of soft robots interacting with the environment," 2018.

[32] E. H. Skorina, M. Luo, W. Tao, F. Chen, J. Fu, and C. D. Onal, "Adapting to flexibility: Model reference adaptive control of soft bending actuators," IEEE Robotics and Automation Letters, vol. 2, no. 2, pp. 964-970, 2017.

[33] C. Keplinger, T. Li, R. Baumgartner, Z. Suo, and S. Bauer, "Harnessing snap-through instability in soft dielectrics to achieve giant voltage-triggered deformation," Soft Matter, vol. 8, no. 2, pp. 285-288, 2012.

[34] I. A. Anderson, T. A. Gisby, T. G. McKay, B. M. O’Brien, and E. P. Calius, "Multi-functional dielectric elastomer artificial muscles for soft and smart machines," Journal of Applied Physics, vol. 112, no. 4, p. 041101, 2012.

[35] J. Overvelde, T. Kloek, J. D'haen, and K. Bertoldi, "Amplifying the response of soft actuators by harnessing snap-through instabilities," Proceedings of the National Academy of Sciences, vol. 112, no. 35, pp. 10863-10868, 2015.

[36] M. Loepfe, C. Schumacher, U. Lustenberger, and W. Stark, "An untethered, jumping roly-poly soft robot driven by combustion," Soft Robotics, vol. 2, no. 1, pp. 33-41, 2015.

[37] W. Felt, K. Chin, and C. Remy, "Contraction sensing with smart braid mckibben muscles," IEEE/ASME Transactions on Mechatronics, vol. 21, no. 3, pp. 1201-1209, 2016.

[38] Y.-L. Park, B.-R. Chen, C. Majidi, R. Wood, R. Nagpal, and E. Goldfield, "Active modular elastomer sleeve for soft wearable assistance robots," in Intelligent Robots and Systems (IROS), 2012 IEEE/RSJ International Conference on, pp. 1595-1602, IEEE, 2012.

[39] Y.-L. Park, C. Majidi, R. Kramer, P. Bérard, and R. Wood, "Hyperelastic pressure sensing with a liquid-embedded elastomer," Journal of Micromechanics and Microengineering, vol. 20, no. 12, p. 125029, 2010.

[40] A. Veale, I. Anderson, and S. Xie, "The smart peano fluidic muscle: a low profile flexible orthosis actuator that feels pain," in SPIE Smart Structures and Materials, pp. 94351V-94351V, International Society for Optics and Photonics, 2015.

[41] H. Lin, F. Guo, F. Wang, and Y.-B. Jia, "Picking up a soft 3d object by "feeling" the grip," The International Journal of Robotics Research, vol. 34, no. 11, pp. 1361-1384, 2015.
[42] X. Luo, H. Wu, H. Yuan, and M. Zhou, "Temporal pattern-aware qos prediction via biased non-negative latent factorization of tensors," IEEE transactions on cybernetics, 2019.

[43] I. Galiana, F. Hammond, R. Howe, and M. Popovic, "Wearable soft robotic device for post-stroke shoulder rehabilitation: Identifying misalignments," in Intelligent Robots and Systems (IROS), 2012 IEEE/RSJ International Conference on, pp. 317-322, IEEE, 2012.

[44] M. Zhu, W. Xu, and L. K. Cheng, "Esophageal peristaltic control of a soft-bodied swallowing robot by the central pattern generator," IEEE/ASME Transactions on Mechatronics, vol. 22, no. 1, pp. 91-98, 2017.

[45] H. In, U. Jeong, H. Lee, and K.-J. Cho, "A novel slack-enabling tendon drive that improves efficiency, size, and safety in soft wearable robots," IEEE/ASME Transactions on Mechatronics, vol. 22, no. 1, pp. 59-70, 2017.

[46] X. Luo, M. Zhou, Y. Xia, Q. Zhu, A. C. Ammari, and A. Alabdulwahab, "Generating highly accurate predictions for missing qos data via aggregating nonnegative latent factor models," IEEE transactions on neural networks and learning systems, vol. 27, no. 3, pp. 524-537, 2015.

[47] X. Luo, M. Zhou, Y. Xia, and Q. Zhu, "An efficient non-negative matrix-factorization-based approach to collaborative filtering for recommender systems," IEEE Transactions on Industrial Informatics, vol. 10, no. 2, pp. 1273-1284, 2014

[48] Y. Hao, Z. Gong, Z. Xie, S. Guan, X. Yang, Z. Ren, T. Wang, and $\mathrm{L}$. Wen, "Universal soft pneumatic robotic gripper with variable effective length," in Control Conference (CCC), 2016 35th Chinese, pp. 6109-6114, IEEE, 2016.

[49] Z. Bingul and O. Karahan, "Comparison of pid and fopid controllers tuned by pso and abc algorithms for unstable and integrating systems with time delay," Optimal Control Applications and Methods, vol. 39, no. 4, pp. 1431-1450, 2018.

[50] Z. Bingul and O. Karahan, "A novel performance criterion approach to optimum design of pid controller using cuckoo search algorithm for avr system," Journal of the Franklin Institute, vol. 355, no. 13, pp. 5534-5559, 2018.

[51] J. T. Agee, Z. Bingul, and S. Kizir, "Tip trajectory control of a flexible-link manipulator using an intelligent proportional integral (ipi) controller," Transactions of the Institute of Measurement and Control, vol. 36, no. 5, pp. 673-682, 2014.

[52] S. J. Wright, "Coordinate descent algorithms," Mathematical Programming, vol. 151, no. 1, pp. 3-34, 2015.

[53] D. Holland, E. Park, P. Polygerinos, G. Bennett, and C. Walsh, "The soft robotics toolkit: Shared resources for research and design," Soft Robotics, vol. 1, no. 3, pp. 224-230, 2014.

[54] "Smooth-on inc." https://www.smooth-on.com/tb/files/DRAGON_SKIN _SERIES_TB.pdf. Accessed: 2018-08-28. 